\title{
Cultivo orgânico de cultivares de café a pleno sol e sombreado
}

\author{
Marta dos Santos Freire Ricci(1), Janaina Ribeiro $\operatorname{Costa}^{(1)}$, Alexandre Nogueira Pinto ${ }^{(1)}$ \\ e Vera Lúcia da Silva Santos ${ }^{(1)}$
}

(1)Embrapa Agrobiologia, Caixa Postal 74505, CEP 23851-970 Seropédica, RJ. E-mail: marta@cnpab.embrapa.br, janaina@cnpab.embrapa.br,
oale@bol.com.br, veralusan@hotmail.com

Resumo - O objetivo deste trabalho foi avaliar a influência de dois sistemas de cultivo orgânico sobre crescimento, fenologia, nutrição e produtividade de cultivares de cafeeiro, e sobre as características químicas do solo. Seis cultivares de café foram avaliadas em monocultura a pleno sol e em cultivo sombreado com banana e Erythrina verna, em delineamento de blocos ao acaso, com quatro repetições. O sombreamento reduziu a taxa de crescimento das cultivares nos primeiros 15 meses de cultivo. Depois de três anos reduziu, também, o diâmetro, o número de ramos produtivos e de nós dos cafeeiros; aumentou a área foliar e o tamanho dos grãos; reduziu o teor de K do solo, e aumentou os teores de $\mathrm{N}$ e de Mg nas folhas do café. Na primeira etapa da colheita (março/2004), a produtividade média das cultivares foi menor no sistema sombreado, o que evidencia que o sombreamento retarda a maturação dos grãos. Na segunda etapa (junho/2004), houve diferença entre cultivares, porém não entre os sistemas de cultivo. A produtividade média total (soma das duas etapas) foi igual nos dois sistemas de cultivo. As cultivares Tupi, Icatu e Obatã são as mais promissoras para o cultivo sombreado.

Termos para indexação: Coffea arabica, Musa sp., Erythrina verna, produtividade, produção orgânica, sombreamento.

\section{Organic cultivation of coffee cultivars grown under full sun and under shading}

\begin{abstract}
The objective of this work was to evaluate the influence of two organic cultivation systems on the growth, phenology, nutrition, and productivity of coffee cultivars, as well as on soil chemical characteristics. Six coffee cultivars were evaluated under both a monoculture system under full sun, and an intercropped system with banana and Erythrina verna plants for shading. The experimental design adopted was the randomized blocks with four replicates. The shading reduced the growth rate of cultivars only during the first 15 months of cultivation. After three years of cultivation, the shading reduced the plant diameter, the number of branches and the number of nodes; increased the leaf area and grain size; reduced soil $\mathrm{K}$ content and increased $\mathrm{N}$ and $\mathrm{Mg}$ contents in coffee plant leaves. In the first harvesting (March 2004), the average yield of the cultivars was smaller in the shading system showing that this treatment delayed grain maturity. In the second harvesting (June 2004), there were differences among cultivars, but not between cropping systems. Yield total average for the two harvestings was the same in the two systems. The cultivars Tupi, Icatu, and Obatã are more promising in shading system.
\end{abstract}

Index terms: Coffea arabica, Musa sp., Erythrina verna, productivity, organic production, shading.

\section{Introdução}

O café é originário de florestas caducifólias da Etiópia e Sudão (Boulay et al., 2000); é, portanto, uma espécie adaptada à sombra. Em vários países produtores de café, tais como Colômbia, Venezuela, Costa Rica, Panamá e México, o cultivo em sistemas agroflorestais (SAF) tem sido um recurso utilizado para aumentar a diversidade vegetal dos sistemas e a renda do produtor (Beer, 1997; Escalante, 1997; Bertrand \& Rapidel, 1999).
No Brasil, a maioria dos produtores prefere o cultivo a pleno sol por acreditarem que o sombreamento diminui a produtividade e porque o cultivo sombreado representa maior necessidade de mão-de-obra, além da dificuldade na passagem de máquinas. Estima-se que mais de $90 \%$ das lavouras existentes são a pleno sol (Ricci et al., 2002).

As informações existentes na literatura, sobre o efeito do sombreamento na produção de café, são escassas e variam de acordo com as condições regionais (altitu- 
de, temperatura, radiação fotossinteticamente ativa e fertilidade do solo) e com a espécie utilizada (Estívariz, 1997). Em muitos estudos conduzidos sobre arborização de cafezais, a sombra reduziu a produção (Hernández et al., 1997). Entretanto, a maioria desses estudos foi conduzida sob sombra muito densa.

Segundo Fernandes (1986), o sombreamento com espécies e espaçamentos adequados pode apresentar resultados satisfatórios, quando comparado ao cultivo a pleno sol. Em relação ao solo, a presença de árvores aumenta o aporte de matéria orgânica em virtude da queda de folhas, conserva a umidade, reduz as perdas de $\mathrm{N}$, aumenta a capacidade de absorção e infiltração de água, reduz o risco de erosão e a emergência de plantas invasoras, e estimula a atividade biológica (Muschler, 2000; Barbera-Castillo, 2001). Adicionalmente, as árvores contribuem para melhorar a fertilidade do solo (Muñoz \& Alvarado, 1997), funcionam como banco de estoque de carbono no solo e na vegetação, removendo quantidades significativas de $\mathrm{CO}_{2}$ da atmosfera (Andrade \& Ibrahim, 2003), e servem como refúgio para a biodiversidade animal (Perfecto et al., 1996; Gormley \& Sinclair, 2003).

A existência de maior biodiversidade possibilita a autorregulação dos sistemas (Dubois, 2004) e proporciona condições desfavoráveis ao estabelecimento de pragas e doenças (Guharay et al., 2001), como por exemplo, a cercosporiose (Cercospora coffeicola), a antracnose (Colletotrichum spp.) (Boulay et al., 2000) e o bicho-mineiro (Leucoptera coffeella), que preferem ambientes ensolarados e mais secos (Haggar et al., 2001).

A produção orgânica é baseada em princípios de diversificação, reciclagem, processos biológicos e imitação de habitats naturais (Ifoam, 1996) e pode ser uma alternativa interessante sob os aspectos ambiental, social e econômico, pois não contamina o meio ambiente nem as pessoas direta e indiretamente envolvidas, além de agregar mais valor ao produto final.

O Brasil é o maior produtor mundial de café; contudo, em 2003, foi o 6opodutor mundial de café orgânico, com uma produção de 80 mil sacas, o que representou 0,2\% de sua produção total (Moreira, 2003). O cultivo orgânico do café em associação com árvores vem sendo estimulado pelas certificadoras, porém não constitui uma condição limitante para a conversão de cafezais convencionais em orgânicos.

O objetivo deste trabalho foi avaliar o efeito de dois sistemas de cultivo orgânico sobre o crescimento, fenologia, nutrição e produtividade de cultivares de café Arábica e sobre as características químicas do solo.

\section{Material e Métodos}

O trabalho foi implantado em janeiro de 2001, em área localizada na Fazenda Santa Mônica, da Embrapa Gado de Leite, em Juparanã, Distrito de Valença, RJ. A área apresenta topografia plana e altitude de $608 \mathrm{~m}$. A precipitação e a temperatura média anual dos últimos dez anos foram, respectivamente, $1.280 \mathrm{~mm}$ e $25,5^{\circ} \mathrm{C}$. O solo analisado (Embrapa, 1997) apresentou as seguintes características químicas: pH, 5,3 (em água); Al, $0,1 \mathrm{cmol}_{\mathrm{C}} \mathrm{dm}^{-3}$; Ca, 1,8 $\mathrm{cmol}_{\mathrm{C}} \mathrm{dm}^{-3} ; \mathrm{Mg}, 0,7 \mathrm{cmol}_{\mathrm{C}} \mathrm{dm}^{-3}$; $\mathrm{P}, 2,0 \mathrm{mg} \mathrm{dm}^{-3}$; K, $128 \mathrm{mg} \mathrm{dm}^{-3}$; carbono orgânico, 13,5 $\mathrm{g} \mathrm{kg}^{-1}$; saturação de bases (V\%), 56,5\%.

Os tratamentos foram dispostos em delineamento de blocos ao acaso, com quatro repetições, em esquema de parcelas subdivididas. Nas parcelas, foram avaliados dois sistemas de manejo (sombreado e a pleno sol) e, nas subparcelas, as seis cultivares de café (Coffea arabica). As médias foram comparadas pelo teste de Scott-Knott a 5\% de probabilidade.

As cultivares de café avaliadas foram: Tupi IAC 1669-33 -, que apresenta porte baixo, maturação precoce, boa produtividade e alta resistência à ferrugem; Oeiras - MG 6851 -, de porte baixo, maturação precoce, produtividade média e resistência à ferrugem de moderada a alta; Icatu amarelo - IAC 3282 -, de porte alto, maturação precoce a tardia, alta produtividade e moderada resistência à ferrugem; Catucaí amarelo 2SL -, de porte baixo a alto, maturação variável, alta produtividade, e moderada a alta resistência à ferrugem; Obatã - IAC 1669-20 -, de porte baixo, maturação tardia, alta produtividade e alta resistência à ferrugem; e Catuaí vermelho - IAC 144 -, que apresenta porte alto, maturação precoce a tardia, alta produtividade e não é resistente à ferrugem.

As mudas foram plantadas em fevereiro de 2001, em parcelas de 27x10 m, constituídas por quatro linhas de cafeeiros, no espaçamento 2,5x0,7 m.

O solo foi preparado e corrigido com $500 \mathrm{~kg} \mathrm{ha}^{-1}$ de calcário dolomítico. As covas de plantio do café foram adubadas com 2,5 kg de esterco de gado (1,67\% de $\mathrm{N}$ ) e $300 \mathrm{~g}$ da mistura de termofosfato magnesiano (18\% de $\mathrm{P}_{2} \mathrm{O}_{5} ; 20 \%$ de $\mathrm{Ca} ; 7,0 \%$ de $\mathrm{Mg}$ ) e cinza de madeira (1:1). Depois de 40 dias, foi feita adubação de cobertura com 250 g de esterco de cama de aviário por planta (2,72\% de $\mathrm{N})$.

Em janeiro de 2002, na área destinada ao cultivo sombreado, foram plantadas mudas de banana (Musa sp. var. Prata Comum) e eritrina (Erythrina verna). 
As mudas de banana foram plantadas nas duas entrelinhas mais externas da parcela, a uma distância de $5 \mathrm{~m}$ entre si, e a cada $3 \mathrm{~m}$ de distância na linha, o que resultou no espaçamento $5 \times 3 \mathrm{~m}$. As covas foram adubadas com $10 \mathrm{~L}$ de esterco de gado e $300 \mathrm{~g}$ da mistura de termofosfato magnesiano. Na mesma época, foram plantadas as mudas de eritrina, distribuídas na mesma linha da banana, a cada $9 \mathrm{~m}$ (espaçamento 5x9 m), tendo recebido a mesma adubação da banana.

A primeira adubação de manutenção constituiu-se de duas aplicações de $250 \mathrm{~g}$ de esterco de cama de aviário e $100 \mathrm{~g}$ de termofosfato magnesiano ( $17 \%$ de $\mathrm{P}_{2} \mathrm{O}_{5}$ solúvel em ácido cítrico; $15 \%$ de $\mathrm{Ca} ; 2 \%$ de $\mathrm{Mg}$ ) por planta, aplicados em outubro de 2001 e março de 2002. Em 2002/2003, a adubação constituiu-se de uma única aplicação de $1 \mathrm{~kg}$ de esterco de cama de aviário e $300 \mathrm{~g}$ de farinha de ossos ( $20 \%$ de $\mathrm{P}_{2} \mathrm{O}_{5}$ solúvel em ácido cítrico; $1,5 \%$ de N; $22 \%$ de Ca) por planta, realizada em fevereiro de 2003. Em outubro de 2003 e em maio de 2004 foram aplicados $3 \mathrm{~kg}$ de composto orgânico (1,37\% de N). O controle das plantas espontâneas foi feito roçando-se as entrelinhas de plantio com roçadeira costal e capinando-se as linhas de plantio com enxada.

O crescimento das cultivares foi avaliado tomando-se dados de altura de dez cafeeiros, selecionados ao acaso na parcela útil, nas seguintes datas: outubro de 2001, maio de 2002, abril e novembro de 2003 e junho de 2004. Nesta última data, avaliou-se o diâmetro do caule à meia altura da planta, a área foliar (amostra de dez folhas-índice), a massa seca das folhas, e os números de ramos produtivos, de nós por ramo e de folhas presentes nos ramos, com base em cinco plantas úteis tomadas ao acaso.

A partir dos dados de altura, tomados nas diferentes épocas de amostragem, calculou-se a taxa de crescimento, utilizando-se a seguinte expressão: taxa de crescimento $=($ altura final - altura inicial $) \times 100 /$ altura inicial.

O número de ramos produtivos foi estimado pela contagem de todos os ramos presentes, do meio da planta até a extremidade. Para a contagem do número de nós, foram selecionados os dois primeiros ramos do terço superior da planta, contando-se o número de folhas presentes (NFP) nesses ramos, de onde foram coletadas dez folhas, as quais foram pesadas e tiveram sua área foliar determinada por meio de um medidor LI-COR 3100. A fim de avaliar o estresse sofrido pelas diferentes cultivares depois da colheita, calculou-se a porcentagem de desfolhamento, considerando-se que o número de folhas total (NFT) deve ser o dobro do número de nós existentes. O número de folhas ausentes (NFA) foi calculado pela diferença entre o NFT e o NFP. A porcentagem de desfolhamento foi calculada a partir do NFT e do NFA, nos dois ramos selecionados para a contagem do NFP, considerando-se a seguinte expressão: \%Desf. $=$ NFAx100/NFT.

Em outubro de 2003, antes da adubação de manutenção, foram retiradas amostras de solos e de folhas. $\mathrm{O}$ solo foi coletado na camada de $0-20 \mathrm{~cm}$ e analisado quanto aos valores de $\mathrm{pH}$ em água, teores de $\mathrm{P}, \mathrm{K}, \mathrm{Ca}, \mathrm{Mg}$ e carbono orgânico (Embrapa, 1997). Amostras de folhas foram coletadas de dez plantas úteis, selecionadas ao acaso, retirando-se o $3^{\circ}$ ou $4^{\circ}$ par de folhas, a partir da extremidade dos ramos situados à meia-altura (Malavolta et al., 1989), e avaliadas quanto aos teores de N, P, K, Ca e Mg. A determinação do teor de $\mathrm{N}$ foi feita a partir da digestão sulfúrica e destilação (Bremner \& Mulvaney, 1982); de P, K, Ca e Mg, por digestão nítrico-perclórica (Bataglia et al., 1983), sendo o P determinado em espectrofotômetro, na faixa visível a partir da formação da cor azul do complexo fosfato-molibdato, em presença de ácido ascórbico como redutor (Embrapa, 1997), e os demais nutrientes, em espectrofotômetro de absorção atômica.

A colheita foi realizada em duas etapas, nos meses de março e junho de 2004, tendo-se coletado somente os frutos maduros ou 'cereja'. A produtividade foi estimada com base em dez plantas úteis, selecionadas ao acaso, e expressa em sacas beneficiadas por hectare. Depois do beneficiamento, obteve-se a massa de 100 grãos.

As variáveis: produção da $1^{\mathrm{a}}$ e da $2^{\mathrm{a}}$ etapa da colheita e diâmetro do caule foram transformadas em, respectivamente, Log (Prod1+1), Log (Prod2) e Log (Diâmetro), por não atenderem às pressuposições da análise de variância, como normalidade e homogeneidade dos erros do modelo.

\section{Resultados e Discussão}

Na primeira avaliação de altura das cultivares, realizada oito meses depois do plantio (outubro de 2001), observaram-se diferenças entre as cultivares: Icatu apresentou maior altura, seguida das cultivares Catucaí, Catuaí vermelho e Oeiras (Tabela 1). Na avaliação seguinte (maio de 2002), quando já havia sombreamento, as cultivares expressaram diferenças de altura, em re- 
lação aos sistemas de cultivo. As cultivares mais altas no sistema a pleno sol foram Catuaí vermelho, Icatu, Catucaí e Oeiras, e no sistema sombreado foram as cultivares Icatu, Catucaí e Catuaí vermelho. A altura média das cultivares, quando sombreadas, foi significativamente menor, sendo a Icatu a que expressou melhor esta diferença. Nos demais períodos avaliados, foram observadas diferenças em altura entre as cultivares, porém não entre os dois sistemas de cultivo.

Na segunda avaliação, a altura média das cultivares foi menor no sistema sombreado (Tabela 1). Nas avaliações seguintes, embora esta tendência tenha permanecido, a diferença não foi significativa. Considerando-se pequena a sombra proporcionada pelas espécies arbóreas no início do ensaio, a menor altura observada pode ser atribuída à competição entre as espécies sombreadoras e o café, por água e nutrientes, visto que inicialmente os sistemas radiculares das espécies sombreadoras e do café ainda encontravam-se superficiais. As menores alturas, obtidas na segunda avaliação (maio/2002), coincidiram com a menor taxa de crescimento (TC\%) das cultivares observada no primeiro período avaliado (outubro/2001 a maio/2002); nesse período de intenso crescimento, a TC média das cultivares foi menor no sistema sombreado (86,6\%), quando comparada ao cultivo a pleno sol $(107,9 \%)$ (Tabela 1$)$.
Nas avaliações seguintes, o sombreamento não afetou a taxa de crescimento dos cafeeiros, em relação ao cultivo a pleno sol, tendo sido observadas somente diferenças significativas entre cultivares, quando submetidas ao mesmo sistema de cultivo, evidenciando as características específicas de cada genótipo.

Os dados de altura e taxas de crescimento, observados durante os 3,5 anos da cultura, demonstraram que as espécies sombreadoras não interferiram, significativamente, no padrão de crescimento das cultivares.

O sombreamento reduziu o diâmetro médio dos cafeeiros, porém não foram observadas diferenças entre as cultivares, nos dois sistemas de cultivo (Tabela 2). A redução do diâmetro médio das cultivares, quando sombreadas, foi acompanhada também pela redução do número médio de ramos produtivos e do número de nós por ramo; as cultivares Catucaí, Obatã e Catuaí vermelho apresentaram maior redução dessas variáveis. Com exceção de Icatu, as cultivares apresentaram menor número de nós por ramo quando sombreadas. A redução do número de ramos produtivos e de nós por ramo já havia sido relatada por outros autores (Estívariz, 1997; Morais et al., 2003) e relacionada à menor atividade fotossintética, ou seja, a valores mais baixos de radiação fotossinteticamente ativa.

Tabela 1. Altura (cm) e taxa de crescimento (\%) das cultivares de café, em razão da época de avaliação e dos sistemas de cultivo, a pleno sol (PS) e sombreado (S) ${ }^{(1)}$.

\begin{tabular}{|c|c|c|c|c|c|c|c|c|c|c|c|c|c|c|c|c|}
\hline \multirow{2}{*}{$\begin{array}{l}\text { Avaliação } \\
\text { (mês/ano) }\end{array}$} & \multicolumn{2}{|c|}{ Catucaí } & \multicolumn{2}{|c|}{ Oeiras } & \multicolumn{2}{|c|}{ Tupi } & \multicolumn{2}{|c|}{ Icatu } & \multicolumn{2}{|c|}{ Obatã } & \multicolumn{2}{|c|}{ Catuaí vermelho } & \multicolumn{2}{|c|}{ Média } & \multicolumn{2}{|c|}{ CV $(\%)$} \\
\hline & PS & $\mathrm{S}$ & PS & $\mathrm{S}$ & PS & $\mathrm{S}$ & PS & $\mathrm{S}$ & PS & $\mathrm{S}$ & PS & $\mathrm{S}$ & PS & $\mathrm{S}$ & Sistema & Cult. \\
\hline & \multicolumn{16}{|c|}{ Altura $(\mathrm{cm})$} \\
\hline $10 / 2001$ & $36,7 \mathrm{Ab}$ & $35,2 \mathrm{Ab}$ & $33,5 \mathrm{Ab}$ & $36,2 \mathrm{Ab}$ & $29,2 \mathrm{Ac}$ & $30,0 \mathrm{Ac}$ & $49,1 \mathrm{Aa}$ & 49,3Aa & $29,0 \mathrm{Ac}$ & $26,7 \mathrm{Ac}$ & $35,4 \mathrm{Ab}$ & $34,4 \mathrm{Ab}$ & $35,5 \mathrm{~A}$ & $35,3 \mathrm{~A}$ & 17,2 & 10,1 \\
\hline $05 / 2$ & $74,4 \mathrm{Aa}$ & $69,2 \mathrm{Aa}$ & $68,5 \mathrm{Aa}$ & $63,6 \mathrm{Ab}$ & $61,0 \mathrm{Ab}$ & $55,7 \mathrm{Ab}$ & $88,7 \mathrm{Aa}$ & $74,9 \mathrm{Ba}$ & $65,9 \mathrm{Ab}$ & $56,2 \mathrm{Ab}$ & $79,0 \mathrm{Aa}$ & $66,7 \mathrm{Aa}$ & $72,9 \mathrm{~A}$ & $64,4 \mathrm{~B}$ & 9,8 & 13,0 \\
\hline $04 / 200$ & $98,4 \mathrm{Ab}$ & $99,7 \mathrm{Ab}$ & $98,1 \mathrm{Ab}$ & $104,0 \mathrm{Ac}$ & $92,6 \mathrm{Ab}$ & $87,3 \mathrm{Ab}$ & $132,9 \mathrm{Aa}$ & $138,7 \mathrm{Aa}$ & $98,7 \mathrm{Ab}$ & $88,6 \mathrm{Ac}$ & $107,5 \mathrm{Ab}$ & $124,8 \mathrm{Ab}$ & $105,5 \mathrm{~A}$ & $104,3 \mathrm{~A}$ & 20,7 & 9,5 \\
\hline $11 / 2$ & $109,8 \mathrm{Ab}$ & $120,8 \mathrm{Ab}$ & $111,8 \mathrm{Ab}$ & $124,5 \mathrm{Ac}$ & $105,5 \mathrm{Ab}$ & $106,9 \mathrm{Ab}$ & $55,6 \mathrm{Ba}$ & $179,3 \mathrm{Aa}$ & $115,4 \mathrm{Ab}$ & 104, & $124,8 \mathrm{Ab}$ & $123,6 \mathrm{Ab}$ & $120,5 \mathrm{~A}$ & $126,6 \mathrm{~A}$ & 14,4 & 10,3 \\
\hline $06 / 2004$ & $0,9 \mathrm{Ab}$ & $141,3 \mathrm{Ab}$ & $130,9 \mathrm{Ab}$ & $142,4 \mathrm{Ab}$ & $128,4 \mathrm{Ab}$ & $124,60 \mathrm{~A}$ & $176,3 \mathrm{Aa}$ & $198,4 \mathrm{Aa}$ & $134,0 \mathrm{Ab}$ & & $149,3 \mathrm{Ab}$ & $0 \mathrm{Ab}$ & $141,6 \mathrm{~A}$ & & 13,2 & 9,6 \\
\hline Período ( & \multicolumn{16}{|c|}{ Taxa de crescimento $(\%)$} \\
\hline $10 / 200$ & $102,2 \mathrm{Aa}$ & $98,1 \mathrm{Aa}$ & $104,9 \mathrm{Aa}$ & $76,0 \mathrm{Bb}$ & $110,2 \mathrm{Aa}$ & $86,6 \mathrm{Aa}$ & $78,6 \mathrm{Ab}$ & $53,5 \mathrm{Ab}$ & $127,2 \mathrm{Aa}$ & $111,6 \mathrm{Aa}$ & $123,1 \mathrm{Aa}$ & $94,0 \mathrm{Ba}$ & $107,9 \mathrm{~A}$ & $86,6 \mathrm{~B}$ & 16,7 & 18,9 \\
\hline $05 / 2002$ & $32,2 \mathrm{Aa}$ & $45,7 \mathrm{Aa}$ & $44,0 \mathrm{Aa}$ & $63,7 \mathrm{Aa}$ & $52,6 \mathrm{Aa}$ & $56,8 \mathrm{Aa}$ & $54,4 \mathrm{Ba}$ & $85,3 \mathrm{Aa}$ & $50,5 \mathrm{Aa}$ & $42,4 \mathrm{Aa}$ & $61,6 \mathrm{Aa}$ & $11,0 \mathrm{Aa}$ & $46,0 \mathrm{~A}$ & $61,9 \mathrm{~A}$ & 53,3 & 30,3 \\
\hline $04 / 2$ & $1,9 \mathrm{Aa}$ & $21,6 \mathrm{Ab}$ & $14,0 \mathrm{Aa}$ & $19,6 \mathrm{Ab}$ & $14,1 \mathrm{Aa}$ & $22,1 \mathrm{Ab}$ & $16,9 \mathrm{Ba}$ & $29,6 \mathrm{Aa}$ & $17,2 \mathrm{Aa}$ & $18,7 \mathrm{Ab}$ & $11,0 \mathrm{Aa}$ & $15,0 \mathrm{Ab}$ & $14,2 \mathrm{~A}$ & $21,1 \mathrm{~A}$ & 56,5 & 32,8 \\
\hline $11 / 2003$ a $06 / 2004$ & $19,7 \mathrm{Aa}$ & $17,3 \mathrm{Ab}$ & $17,5 \mathrm{Aa}$ & $14,4 \mathrm{Ab}$ & $22,1 \mathrm{Aa}$ & $17,0 \mathrm{Ab}$ & $13,7 \mathrm{Aa}$ & $10,5 \mathrm{Ab}$ & $16,3 \mathrm{Ba}$ & $27,0 \mathrm{Aa}$ & $19,7 \mathrm{Aa}$ & $23,3 \mathrm{Aa}$ & $18,2 \mathrm{~A}$ & $18,3 \mathrm{~A}$ & 31,9 & 40,7 \\
\hline
\end{tabular}

(1)Médias seguidas de letras iguais, maiúsculas (sistema de manejo) e minúsculas (cultivares) na linha, não diferem significativamente pelo teste de Scott \& Knott $(1974)(\mathrm{p}<0,05)$.

Tabela 2. Diâmetro do caule, número de ramos primários, número de nós por ramo, área foliar, massa seca de folhas e desfolhamento das cultivares de café, em razão dos sistemas de cultivo, a pleno sol (PS) e sombreado (S), em agosto de 2004(1).

\begin{tabular}{|c|c|c|c|c|c|c|c|c|c|c|c|c|c|c|c|c|}
\hline \multirow[t]{2}{*}{ Variável } & \multicolumn{2}{|c|}{ Catucaí } & \multicolumn{2}{|c|}{ Oeiras } & \multicolumn{2}{|c|}{ Tupi } & \multicolumn{2}{|c|}{ Icatu } & \multicolumn{2}{|c|}{ Obatã } & \multicolumn{2}{|c|}{ Catuaí vermelho } & \multicolumn{2}{|c|}{ Média } & \multicolumn{2}{|c|}{ CV (\%) } \\
\hline & PS & $\mathrm{S}$ & PS & $\mathrm{S}$ & PS & $\mathrm{S}$ & PS & $\mathrm{S}$ & PS & $\mathrm{S}$ & PS & $\mathrm{S}$ & PS & $\mathrm{S}$ & Sistema & Cult. \\
\hline Diân & $1,93 \mathrm{Aa}$ & $1,67 \mathrm{Ba}$ & $1,94 \mathrm{Aa}$ & $1,78 \mathrm{Aa}$ & $2,13 \mathrm{Aa}$ & $1,88 \mathrm{Ba}$ & $2,12 \mathrm{Aa}$ & $1,80 \mathrm{Ba}$ & $2,02 \mathrm{Aa}$ & $1,69 \mathrm{Ba}$ & $2,02 \mathrm{Aa}$ & $1,69 \mathrm{Ba}$ & $2,02 \mathrm{~A}$ & $1,75 \mathrm{~B}$ & 12,3 & 12,3 \\
\hline № de r & $9,4 \mathrm{Aa}$ & $15,2 \mathrm{Ba}$ & $17,5 \mathrm{Ab}$ & $15,7 \mathrm{Aa}$ & $15,8 \mathrm{Ab}$ & $13,8 \mathrm{Aa}$ & $18,2 \mathrm{Ab}$ & $16,4 \mathrm{Aa}$ & $8,8 \mathrm{Aa}$ & $15,2 \mathrm{Ba}$ & $21,2 \mathrm{Aa}$ & $16,5 \mathrm{Ba}$ & $18,5 \mathrm{~A}$ & $15,5 \mathrm{~B}$ & 10,3 & 10,7 \\
\hline № de $n$ & 9Ab & $11,0 \mathrm{Ba}$ & $11,8 \mathrm{Ab}$ & $9,60 \mathrm{Bb}$ & $12,8 \mathrm{Ab}$ & $10,5 \mathrm{Bb}$ & $14,2 \mathrm{Aa}$ & $12,7 \mathrm{Aa}$ & 11,5 & $9,0 \mathrm{Bb}$ & $\mathrm{Aa}$ & $11,3 \mathrm{Ba}$ & $12,9 \mathrm{~A}$ & 10,7 & 8,0 & 9,8 \\
\hline $\operatorname{ar}\left(\mathrm{cm}^{2}\right)^{(3)}$ & $2 \mathrm{Aa}$ & $384 \mathrm{Bc}$ & $506 \mathrm{Aa}$ & $458 \mathrm{Ab}$ & $537 \mathrm{Aa}$ & $466 \mathrm{Ab}$ & $422 \mathrm{Ab}$ & $338 \mathrm{Ac}$ & $594 \mathrm{Aa}$ & $580 \mathrm{Aa}$ & $468 \mathrm{Ab}$ & $400 \mathrm{Ac}$ & $438 \mathrm{~B}$ & $508 \mathrm{~A}$ & 12,3 & 12,3 \\
\hline Massa sec & $35 \mathrm{Bb}$ & $5,83 \mathrm{Aa}$ & $5,14 \mathrm{Ab}$ & $5,46 \mathrm{Aa}$ & $5,75 \mathrm{Aa}$ & $6,12 \mathrm{Aa}$ & $4,39 \mathrm{Ab}$ & $4,48 \mathrm{Ab}$ & $6,62 \mathrm{Aa}$ & $6,25 \mathrm{Aa}$ & $4,80 \mathrm{Ab}$ & $4,79 \mathrm{Ab}$ & $5,17 \mathrm{~A}$ & $5,49 \mathrm{~A}$ & 7,4 & 8,4 \\
\hline Desfolhamento (\%) & $66,7 \mathrm{Aa}$ & $42,6 \mathrm{Ba}$ & $55,2 \mathrm{Aa}$ & $38,5 \mathrm{Ba}$ & $60,3 \mathrm{Aa}$ & $32,4 \mathrm{Ba}$ & $57,4 \mathrm{Aa}$ & $42,6 \mathrm{Aa}$ & $68,7 \mathrm{Aa}$ & $43,4 \mathrm{Ba}$ & $68,0 \mathrm{Aa}$ & $45,7 \mathrm{Ba}$ & $62,7 \mathrm{~A}$ & $40,9 \mathrm{~B}$ & 28,7 & 17,6 \\
\hline
\end{tabular}

(1)Médias seguidas de letras iguais, maiúsculas (sistema de manejo) e minúsculas (cultivares) na linha, não diferem significativamente pelo teste de Scott \& Knott (1974) $(\mathrm{p}<0,05) .{ }^{(2)}$ Número de ramos produtivos, contados da metade da planta para cima. (3)Área foliar total de dez folhas. 
O sombreamento proporcionou maiores valores de área foliar, não tendo alterado, porém, a massa média das folhas (Tabela 2). A expansão da área foliar também foi observada por Morais et al. (2003) e pode ser um mecanismo utilizado pelo cafeeiro para compensar a menor luminosidade recebida, quando sombreado. Segundo Cannell (1985), o aumento da área foliar por unidade de área é um dos fatores que concorrem para aumentar a produção.

Uma avaliação realizada no mês de agosto $(0,5 \mathrm{~mm}$ de precipitação) revelou que o sombreamento reduziu o desfolhamento médio das cultivares (Tabela 2). O sombreamento propicia uma melhor condição climática ao cafeeiro, protege-o do estresse provocado por elevadas temperaturas e perda de umidade do solo, reduz a evapotranspiração (Boulay et al., 2000), e evita, desta forma, um maior desfolhamento durante a estação seca. Em relação à influência dos sistemas de cultivo sobre as características químicas do solo, no terceiro ano de cultivo, uma análise realizada em novembro de 2003 revelou que o teor de $\mathrm{K}$ foi significativamente menor no sistema sombreado (112 $\mathrm{mg} \mathrm{dm}^{-3}$ ), quando comparado ao sistema a pleno sol (216 $\mathrm{mg} \mathrm{dm}^{-3}$ ), não tendo havido diferença entre os sistemas quanto às características pH, P, Ca, Mg e carbono orgânico (Tabela 3). A diferença observada no teor de $\mathrm{K}$ pode ser explicada pelo fato de a bananeira associada ao café ser uma espécie exigen-

Tabela 3. Valores médios de pH, P, K, Ca, Mg e carbono orgânico do solo (CO), em razão dos sistemas de cultivo, em novembro de $2003^{(1)}$.

\begin{tabular}{lcccccc}
\hline Sistema de manejo & $\begin{array}{c}\mathrm{pH} \\
\left(\mathrm{H}_{2} \mathrm{O}\right)\end{array}$ & $\begin{array}{c}\mathrm{P} \\
---\left(\mathrm{mg} \mathrm{dm}^{-3}\right)---\end{array}$ & $\begin{array}{c}\mathrm{Ca} \\
\left(\mathrm{cmol}_{\mathrm{c}} \mathrm{mg} \mathrm{kg}^{-1}\right)--\end{array}$ & $\mathrm{CO}(\%)$ \\
\hline Café a pleno sol & $6,58 \mathrm{~A}$ & $64,3 \mathrm{~A}$ & $216 \mathrm{~A}$ & $3,03 \mathrm{~A}$ & $1,60 \mathrm{~A}$ & $1,44 \mathrm{~A}$ \\
Café sombreado & $6,31 \mathrm{~A}$ & $70,3 \mathrm{~A}$ & $112 \mathrm{~B}$ & $2,84 \mathrm{~A}$ & $1,95 \mathrm{~A}$ & $1,43 \mathrm{~A}$ \\
\hline Valor inicial no solo & 5,30 & 2,0 & 128 & 1,80 & 0,70 & 1,35 \\
\hline CV (\%) (Sistema) & 7,7 & 26,7 & 49,4 & 8,1 & 28,3 & 7,9 \\
CV (\%) (Cultivar) & 4,5 & 52,9 & 32,6 & 20,1 & 23,3 & 5,8 \\
\hline
\end{tabular}

${ }^{(1)}$ Médias seguidas de letras iguais, na coluna, não diferem significativamente pelo teste de Scott \& Knott (1974) $(\mathrm{p}<0,05)$. te em relação ao K, que acumula nos seus frutos e folhas, respectivamente, 33,7 e $14 \mathrm{~g} \mathrm{~kg}^{-1}$ de K (Espíndola, 2001).

Em relação aos valores encontrados inicialmente no solo, em 2001, observaram-se, em ambos os sistemas, aumento nos valores de $\mathrm{pH}$, bem como nos teores de $\mathrm{P}$, K, Ca, Mg e carbono orgânico (Tabela 3). Os teores elevados de P, em relação ao teor inicial, deve-se ao fato de ter sido utilizada, por dois anos consecutivos, farinha de ossos com $20 \%$ de $\mathrm{P}_{2} \mathrm{O}_{5}$ solúvel em ácido cítrico.

Uma análise realizada em novembro de 2003, no tecido foliar, revelou que, comparativamente ao cultivo a pleno sol, o sombreamento proporcionou ao cafeeiro maiores teores de $\mathrm{N}$ e de $\mathrm{Mg}$ no tecido foliar das cultivares, não tendo alterado os teores de $\mathrm{P}, \mathrm{K} \mathrm{e} \mathrm{Ca}$ (Tabela 4).

Os maiores teores de $\mathrm{N}$, encontrados no tecido foliar dos cafeeiros, confirmam a hipótese de que o sistema sombreado é mais apropriado para o cultivo orgânico do café, tendo-se em vista que o $\mathrm{N}$ é um dos fatores mais limitantes neste sistema de cultivo. Os maiores teores encontrados estão relacionados à presença de uma leguminosa no sistema (eritrina), e às menores perdas de $\mathrm{N}$ do solo no sistema sombreado. A presença de espécies sombreadoras implica maior ciclagem de nutrientes pela queda de folhas e galhos, maior proteção do solo contra erosão, menor exposição do solo à radiação solar e maior presença de raízes que, por sua vez, evitam que o N seja perdido (Muñoz \& Alvarado, 1997), e aumentam a atividade biológica do solo, favorecendo as relações solo-planta e a fixação biológica de $\mathrm{N}_{2}$ (Beer, 1988).

Na primeira etapa da colheita (março de 2004), a produtividade média das cultivares foi maior no sistema a pleno sol, o que demonstra que o sombreamento retarda a maturação dos grãos (Tabela 5). Conforme relatado por Boulay et al. (2000), este é um fator que fa-

Tabela 4. Teores de N, P, K, Ca e Mg ( $\mathrm{g} \mathrm{kg}^{-1}$ ) do tecido foliar das cultivares de café, em razão dos sistemas de cultivo, a pleno sol (PS) e sombreado (S), em novembro de $2003^{(1)}$.

\begin{tabular}{|c|c|c|c|c|c|c|c|c|c|c|}
\hline \multirow[t]{2}{*}{ Cultivares } & \multicolumn{2}{|c|}{$\mathrm{N}$} & \multicolumn{2}{|c|}{$\mathrm{P}$} & \multicolumn{2}{|c|}{$\mathrm{K}$} & \multicolumn{2}{|c|}{$\mathrm{Ca}$} & \multicolumn{2}{|c|}{$\mathrm{Mg}$} \\
\hline & PS & $\mathrm{S}$ & PS & $\mathrm{S}$ & PS & $\mathrm{S}$ & PS & S. & PS & $\mathrm{S}$ \\
\hline Catucaí & $26,4 \mathrm{Aa}$ & $28,3 \mathrm{Aa}$ & $2,15 \mathrm{Aa}$ & $2,01 \mathrm{Aa}$ & $25,1 \mathrm{Aa}$ & $24,8 \mathrm{Aa}$ & $8,3 \mathrm{Aa}$ & $9,8 \mathrm{Aa}$ & $3,19 \mathrm{Aa}$ & $3,38 \mathrm{Ac}$ \\
\hline Oeiras & $28,1 \mathrm{Aa}$ & $28,6 \mathrm{Aa}$ & $1,68 \mathrm{Ab}$ & $1,98 \mathrm{Aa}$ & $25,3 \mathrm{Aa}$ & $23,3 \mathrm{Aa}$ & $9,3 \mathrm{Aa}$ & $10,4 \mathrm{Aa}$ & $3,24 \mathrm{Aa}$ & $3,45 \mathrm{Ac}$ \\
\hline Tupi & $25,6 \mathrm{Ba}$ & $28,8 \mathrm{Aa}$ & $1,58 \mathrm{Ab}$ & $1,85 \mathrm{Aa}$ & $23,8 \mathrm{Aa}$ & $23,8 \mathrm{Aa}$ & $8,5 \mathrm{Aa}$ & $9,2 \mathrm{Aa}$ & $2,86 \mathrm{Aa}$ & $3,01 \mathrm{Ab}$ \\
\hline Icatu & $29,1 \mathrm{Aa}$ & $29,9 \mathrm{Aa}$ & $1,77 \mathrm{Ab}$ & $2,07 \mathrm{Aa}$ & $25,9 \mathrm{Aa}$ & $25,5 \mathrm{Aa}$ & $9,0 \mathrm{Ba}$ & $11,1 \mathrm{Aa}$ & $3,55 \mathrm{Ba}$ & $4,01 \mathrm{Aa}$ \\
\hline Obatã & $24,6 \mathrm{Ba}$ & $27,9 \mathrm{Aa}$ & $1,67 \mathrm{Ab}$ & $1,87 \mathrm{Aa}$ & $24,3 \mathrm{Aa}$ & $25,0 \mathrm{Aa}$ & $9,8 \mathrm{Aa}$ & $10,2 \mathrm{Aa}$ & 3,19Aa & $3,28 \mathrm{Ac}$ \\
\hline Catuaí vermelho & $26,4 \mathrm{Aa}$ & $28,8 \mathrm{Aa}$ & $2,16 \mathrm{Aa}$ & $2,26 \mathrm{Aa}$ & $25,6 \mathrm{Aa}$ & $26,4 \mathrm{Aa}$ & $8,6 \mathrm{Aa}$ & $10,2 \mathrm{Aa}$ & $3,21 \mathrm{Aa}$ & $3,54 \mathrm{Ac}$ \\
\hline Média & $26,7 \mathrm{~B}$ & $28,7 \mathrm{~A}$ & $1,83 \mathrm{~A}$ & $2,00 \mathrm{~A}$ & $24,8 \mathrm{~A}$ & $25,0 \mathrm{~A}$ & $8,9 \mathrm{~A}$ & $10,2 \mathrm{~A}$ & $3,21 \mathrm{~B}$ & $3,44 \mathrm{~A}$ \\
\hline Nível crítico $\left(\mathrm{g} \mathrm{kg}^{-1}\right)$ & \multicolumn{2}{|c|}{30} & \multicolumn{2}{|c|}{$0,8-1,0$} & \multicolumn{2}{|c|}{18} & \multicolumn{2}{|c|}{10} & \multicolumn{2}{|c|}{3,5} \\
\hline CV (\%) (Sistema) & \multicolumn{2}{|c|}{5,3} & \multicolumn{2}{|c|}{25,9} & \multicolumn{2}{|c|}{6,3} & \multicolumn{2}{|c|}{18,3} & \multicolumn{2}{|c|}{5,3} \\
\hline CV (\%) (Cultivar) & \multicolumn{2}{|c|}{6,7} & \multicolumn{2}{|c|}{10,3} & \multicolumn{2}{|c|}{5,6} & \multicolumn{2}{|c|}{9,0} & \multicolumn{2}{|c|}{6,9} \\
\hline
\end{tabular}

(1) Médias seguidas de letras iguais, maiúsculas na linha (manejo) e minúsculas na coluna (cultivares), não diferem significativamente pelo teste de Scott \& Knott (1974) $(\mathrm{p}<0,05)$. 
Tabela 5. Produtividade (sacas de $60 \mathrm{~kg} \mathrm{ha}^{-1}$ ), das cultivares de café na primeira e na segunda etapa da colheita de 2004, e massa (g) de 100 grãos beneficiados, em razão dos sistemas de cultivo, a pleno sol (PS) e sombreado (S) ${ }^{(1)}$.

\begin{tabular}{|c|c|c|c|c|c|c|c|c|}
\hline \multirow[t]{2}{*}{ Cultivares } & \multicolumn{2}{|c|}{$1^{a}$ etapa } & \multicolumn{2}{|c|}{$2^{\mathrm{a}}$ etapa } & \multicolumn{2}{|c|}{ Total } & \multicolumn{2}{|c|}{$\mathrm{P} 100(\mathrm{~g})$} \\
\hline & PS & $\mathrm{S}$ & PS & $\mathrm{S}$ & PS & $\mathrm{S}$ & PS & $\mathrm{S}$ \\
\hline Catucaí & $7,56 \mathrm{Ab}$ & $1,95 \mathrm{Ba}$ & $8,87 \mathrm{Ab}$ & $12,61 \mathrm{Ab}$ & $16,43 \mathrm{Aa}$ & $14,56 \mathrm{Ab}$ & $18,15 \mathrm{Ba}$ & $20,24 \mathrm{Ab}$ \\
\hline Oeiras & $5,18 \mathrm{Ab}$ & $0,85 \mathrm{Ba}$ & $24,96 \mathrm{Aa}$ & $16,53 \mathrm{Ab}$ & $30,14 \mathrm{Aa}$ & $17,37 \mathrm{Ab}$ & $18,18 \mathrm{Ba}$ & $21,15 \mathrm{Aa}$ \\
\hline Tupi & $2,91 \mathrm{Ab}$ & $0,96 \mathrm{Ba}$ & $23,44 \mathrm{Aa}$ & $26,94 \mathrm{Aa}$ & $26,34 \mathrm{Aa}$ & $27,90 \mathrm{Aa}$ & $17,89 \mathrm{Ba}$ & $21,96 \mathrm{Aa}$ \\
\hline Icatu & $9,87 \mathrm{Aa}$ & $5,70 \mathrm{Aa}$ & $10,27 \mathrm{Ab}$ & $22,01 \mathrm{Aa}$ & $20,14 \mathrm{Aa}$ & $27,70 \mathrm{Aa}$ & $16,34 \mathrm{Ba}$ & $19,01 \mathrm{Ab}$ \\
\hline Obatã & $12,04 \mathrm{Aa}$ & $4,08 \mathrm{Ba}$ & $15,84 \mathrm{Ab}$ & $27,26 \mathrm{Aa}$ & $22,87 \mathrm{Aa}$ & $31,33 \mathrm{Aa}$ & $17,74 \mathrm{Ba}$ & $20,26 \mathrm{Ab}$ \\
\hline Catuaí vermelho & $11,41 \mathrm{Aa}$ & $1,52 \mathrm{Ba}$ & $6,10 \mathrm{Ab}$ & $15,10 \mathrm{Ab}$ & $17,51 \mathrm{Aa}$ & $16,63 \mathrm{Ab}$ & $17,64 \mathrm{Ba}$ & $21,60 \mathrm{Aa}$ \\
\hline Média & $8,16 \mathrm{~A}$ & $2,51 \mathrm{~B}$ & $14,91 \mathrm{~A}$ & $20,07 \mathrm{~A}$ & $23,07 \mathrm{~A}$ & $22,58 \mathrm{~A}$ & $17,66 \mathrm{~B}$ & $20,70 \mathrm{~A}$ \\
\hline CV\% (Sistema) & \multicolumn{2}{|c|}{27,4} & \multicolumn{2}{|c|}{29,2} & \multicolumn{2}{|c|}{49,3} & \multicolumn{2}{|c|}{3,8} \\
\hline CV\% (Cultivar) & \multicolumn{2}{|c|}{31,0} & \multicolumn{2}{|c|}{18,4} & \multicolumn{2}{|c|}{43,0} & \multicolumn{2}{|c|}{6,2} \\
\hline
\end{tabular}
Scott \& Knott $(1974)(\mathrm{p}<0,05)$.

vorece a qualidade de bebida do café, além de indicar que a colheita será mais tardia do que em cafezais conduzidos a pleno sol. Na segunda etapa da colheita (junho de 2004), observaram-se diferenças entre as cultivares, porém não entre os sistemas de cultivo.

A produtividade média total (soma das duas etapas) das cultivares não foi reduzida pelo sombreamento. No sistema a pleno sol, não houve diferenças entre as cultivares; porém, quando sombreadas, as cultivares Tupi, Icatu e Obatã foram as mais produtivas (Tabela 5). Embora o sombreamento tenha reduzido o número médio de ramos produtivos e de nós (Tabela 2), os dados de produção obtidos neste trabalho demonstram que o sombreamento utilizado foi adequado às condições ambientais do experimento (altitude, temperatura, fertilidade do solo), confirmando o que outros autores já haviam observado (Moreira, 2003). Outra hipótese que pode explicar os resultados está relacionada à maior conversão de flor em fruto, observada por Estívariz (1997), associada a valores mais baixos de radiação fotossinteticamente ativa.

No sistema sombreado, a massa média de 100 grãos beneficiados foi maior, podendo-se afirmar que o sombreamento aumentou o tamanho dos grãos, refletido em seu aspecto físico, melhorando a classificação por peneira. Tais resultados estão de acordo com os encontrados por outros autores (Estívariz, 1997; Muschler, 2001).

\section{Conclusões}

1. O sombreamento reduz a taxa de crescimento das cultivares apenas nos primeiros 15 meses de crescimento.

2. O sombreamento reduz o diâmetro dos cafeeiros, o número de ramos produtivos e de nós por ramos, mas aumenta a área foliar e o peso dos grãos, permitindo a obtenção de produção semelhante ao cultivo a pleno sol.

3. Depois de três anos, o cultivo do café com bananeiras e eritrinas reduz o teor de $\mathrm{K}$ do solo, em relação ao sistema a pleno sol, e proporciona maiores teores de $\mathrm{N}$ e Mg no tecido foliar do cafeeiro.

4. As cultivares Tupi, Icatu e Obatã são as mais promissoras para serem utilizadas em sistemas sombreados.

5. O sombreamento retarda a maturação dos grãos.

\section{Agradecimentos}

À Embrapa Café, pela concessão de bolsa de apoio técnico a Alexandre Nogueira Pinto e Vera Lúcia da Silva Santos.

\section{Referências}

ANDRADE, H.J.; IBRAHIM, M. ¿Cómo monitorear el secuestro de carbono en los sistemas silvopastoriles? Agroforestería en las Américas, v.10, p.109-116, 2003.

BARBERA-CASTILLO, N.M. Diversidad de especies de hormigas en sistemas agroforestales contrastantes de café, en Turrialba, Costa Rica. 2001. 99p. Dissertação (Mestrado) - Centro Agronómico Tropical de Investigación y Enseñanza, Turrialba, Costa Rica.

BATAGLIA, O.C.; FURLANI, A.M.C.; TEIXEIRA, J.P.F.; FURLANI, P.R.; GALLO, J.R. Método de análise química de plantas. Campinas: Instituto Agronômico, 1983. 48p. (Boletim técnico, 78).

BEER, J. Café bajo sombra en América Central ¿Hace falta más investigación sobre este sistema agroflorestal exitoso? Agroforestería en las Américas, v.4, p.8-13, 1997.

BEER, J. Litter production and nutrient cycling in coffee (Coffea arabica) or cacao (Theobroma cacao) plantations with shade trees. Agroforestry Systems, v.7, p.103-114, 1988. 
BERTRAND, B.; RAPIDEL, B. Desafíos de la caficultura en Centroamérica. San José, Costa Rica: Promecafe; Paris: Cirad, 1999. 496p.

BOULAY, M.; SOMARRIBA, E.; OLIVIER, A. Calidad de Coffea arabica bajo sombra de Erythrina poeppigiana a diferentes elevaciones en Costa Rica. Agroforestería en las Américas, v.7, p.40-42, 2000.

BREMNER, J.M.; MULVANEY, C.S. Nitrogen total. In: KLUTE, A.; PAGE, A.L. (Ed.). Methods of soil analysis. $2^{\text {nd }}$ ed. Madison: American Society of Agronomy, 1982. v.2, p.595-624. (Agronomy, 9).

CANNELL, R. Physiology of the coffee crop. In: CLIFFORD, M.N.; WILLSON, K.C. (Ed.). Coffee: botany, biochemistry and production of beans and beverage. London: Croom Helm, 1985. p.108-134.

DUBOIS, J.C.L. Biodiversificação de SAFs. Rebraf, 2004. Disponível em: <http://www.rebraf.org.br>. Acesso em: 24 ago. 2004.

EMBRAPA. Centro Nacional de Pesquisa de Solos (Rio de Janeiro, RJ). Manual de métodos de análise de solo. 2.ed. Rio de Janeiro, 1997. 212p. (Documentos, 1).

ESCALANTE, E. Café y agroforestería en Venezuela. Agroforestería en las Américas, v.4, p.21-24, 1997.

ESPÍNDOLA, J.A.A. Avaliação de leguminosas herbáceas perenes usadas como cobertura viva do solo e sua influência sobre a produção da bananeira (Musa spp.). 2001. 170p. Tese (Doutorado) - Universidade Federal Rural do Rio de Janeiro, Seropédica, RJ.

ESTÍVARIZ-COCA, J.J. Efecto de sombra sobre la floración y producción de café (Coffea arabica var. Caturra), después de una poda completa en Turrialba, Costa Rica. 1997. 65p. Dissertação (Mestrado) - Centro Agronómico Tropical de Investigación y Enseñanza, Turrialba, Costa Rica.

FERNANDES, D.R. Manejo do cafezal. In: RENA, A.B.; MALAVOLTA, E.; ROCHA, M.; YAMADA, T. (Ed.). Cultura do café; fatores que afetam a produtividade. Piracicaba, SP: Associação Brasileira para Pesquisa da Potassa e do Fosfato, 1986. p.275-301.

GORMLEY, L.H.L.; SINCLAIR, F.L. Modelaje participativo del impacto de los árboles en la productividad de las fincas y la biodiversidad regional en paisajes fragmentados en América Central. Agroforestería en las Américas, v.10, p.103-108, 2003.
GUHARAY, F.; MONTERROSO, D.; STAVER, C. El diseño y manejo de la sombra para la supresión de plagas en cafetales de América Central. Agroforestería en las Américas, v.8, p.22-29, 2001.

HAGGAR, J.; SCHIBLI, C.; STAVER, C. ¿Cómo manejar árboles de sombra en cafetales? Agroforestería en las Américas, v.8, p.4245, 2001.

HERNÁNDEZ, O.; BEER, J.; PLATEN, H. von. Rendimiento de café (Coffea arabica) cv. Caturra, producción de madera (Cordia alliodora) y análisis financiero de plantaciones con diferentes densidades de sombra en Costa Rica. Agroforestería en las Américas, v.4, p.8-13, 1997.

IFOAM. Basic standards for organic agriculture and processing, and guidelines for coffee, cocoa and tea: evaluation of inputs. Tholey-Theley, Germany, 1996. 44p.

MALAVOLTA, E.; VITTI, G.C.; OLIVEIRA, S.A. de. Avaliação do estado nutricional das plantas: princípios e aplicações. Piracicaba: Potafos, 1989. 201p.

MORAIS, H.; MARUR, C.J.; CARAMORI, P.H.; RIBEIRO, A.M. de A.; GOMES, J.C. Características fisiológicas e de crescimento de cafeeiro sombreado com guandu e cultivado a pleno sol. Pesquisa Agropecuária Brasileira, v.38, p.1131-1137, 2003.

MOREIRA, C.F. Caracterização de sistemas de café orgânico sombreado e a pleno sol no sul de Minas Gerais. 2003. 125p. Tese (Mestrado) - Escola Superior de Agricultura Luiz de Queiroz, Piracicaba, SP.

MUÑOZ, G.; ALVARADO, J. Importancia de la sombra en el cafetal. Agroforestería en las Américas, v.4, p.25-29, 1997.

MUSCHLER, R.G. Árboles en cafetales. Turrialba, Costa Rica: Catie/ GTZ, 2000. 139p. (Módulo de enseñanza agroforestal, 5).

MUSCHLER, R.G. Shade improves coffee quality in a sub-optimal coffee-zone of Costa Rica. Agroforestry Systems, v.51, p.131139, 2001.

PERFECTO, I.; RICE, R.A.; GREENBERG, R.; VAN DER VOORT, M.E. Shade coffee: a disappearing refuge for biodiversity. BioScience, v.46, p.598-608, 1996.

RICCI, M. dos S.F.; ARAÚJO, M. do C.F.; FRANCH, C.M. de C. Cultivo orgânico do café: recomendações técnicas. Brasília: Embrapa Informação Tecnológica, 2002. 101p.

SCOTT, A.J.; KNOTT, M.A. A cluster analysis method for grouping means in the analysis of variance. Biometrics, v.30, p.507-512, 1974.

$\overline{\text { Recebido em } 28 \text { de dezembro de } 2004 \text { e aprovado em } 2 \text { de dezembro de } 2005}$ 